\title{
Influencia de los aspectos demográficos en la complejidad tecnológica humana
}

\author{
Influence of demographic aspects on human \\ technological complexity
}

\author{
ÁLVARO MORENO MARÍN \\ Universidad de Málaga
}

Recibido: 12/11/2020 Aceptado: 23/11/2020

\section{RESUMEN}

El distanciamiento temporal entre la aparición de Homo sapiens y la consolidación de los rasgos conductualmente modernos entre las poblaciones humanas plantea el problema de cuál es el factor, o factores, que explican dicha consolidación en un momento particular de nuestra historia: la transición entre EPM/PM y EPS/PS. En este artículo se analizan varias de las propuestas que han tratado de identificar la causa de este cambio cultural, en especial las que relacionan el grado de complejidad tecnológico alcanzado por una población con sus características demográficas.

\section{PALABRAS CLAVE \\ MODERNIDAD CONDUCTUAL, COMPLEJIDAD TECNOLÓGICA, DEMOGRAFÍA, MODELOS DEMOGRÁFICOS}

\begin{abstract}
The lag between the appearance of Homo sapiens and the consolidation of behaviorally modern traits among human populations raises the problem of what is the factor, or factors, that explain this consolidation in a particular moment of our history: the transition between MSA/MP and LSA/UP. This paper examines several of the proposals that have tried to identify the cause of
\end{abstract}

(C) Contrastes. Revista Internacional de Filosofía, vol. XXVI N³ (2021), pp. 149-169. ISSN: 1136-4076

Departamento de Filosofía, Universidad de Málaga, Facultad de Filosofía y Letras

Campus de Teatinos, E-29071 Málaga (España) 
this cultural change, especially those that relate the degree of technological complexity reached by a population with its demographic characteristics.

KEYWORDS

BEHAVIORAL MODERNITY, TECHNOLOGICAL COMPLEXITY, DEMOGRAPHY, DEMOGRAPHIC MODELS

\section{EL PROBLEMA DE LA MODERNIDAD CONDUCTUAL}

HaCe APROXIMADAMENTE 45.000 AÑos tuvo lugar uno de los mayores y más decisivos cambios culturales y tecnológicos de nuestra historia, con unas consecuencias tal vez sólo superadas por la transformación ganadera y agrícola del Neolítico. Por esas fechas, empezó a extenderse y consolidarse entre los Homo sapiens anatómicamente modernos, surgidos hace unos 200.000 años, una forma de comportamiento que marcó sin lugar a dudas un punto de inflexión en nuestra historia como especie. Dicho momento coincide con la consolidación de un nuevo tipo de industria tecnológica que va a dar paso a la Edad de Piedra Superior o Tardía ${ }^{1}$ / Paleolítico Superior ${ }^{2}$ (en adelante EPS/ PS) que reemplazará a la anterior tecnología de la Edad de Piedra Media / Paleolítico Medio (en adelante EPM/PM), en algunos casos de manera rápida y expansiva.

Esta última había comenzado antes de la aparición de los humanos anatómicamente modernos, desplazando a la tecnología achelense unos 250.000 años atrás, diversificando la tipología de las herramientas líticas (que en la tecnología achelense había tenido un claro predominio de las hachas de mano o bifaces) y acentuando el uso de lascas retocadas, cuya morfología muestra un mayor grado de especialización para las diferentes tareas en que eran empleadas.

Sin embargo, aunque la tecnología perteneciente a la cultura de EPM/PM posee una marcada diferencia con la tecnología achelense, se encuentra aún en general bastante alejada de los rasgos que caracterizarán posteriormente a las herramientas asociadas a la modernidad conductual de la tecnología EPS/ PS, debido principalmente a su poca variación espacio-temporal y a su fuerte uniformidad tipológica. La llegada de la tecnología EPS/PS trajo una reducción significativa del tamaño de las herramientas (microlitos), principalmente en forma de hojas delgadas y de filo cortante gracias al lascado por presión. Su morfología comenzó a estar mucho más especializada y a mostrar un alto

1 En el África subsahariana.

2 En el norte de África, Europa, el Levante Mediterráneo y Asia occidental. 
grado de variación. Aparecieron útiles compuestos como arcos y flechas, redes de pesca o propulsores para aumentar la fuerza de lanzamiento de los venablos. Se diversificaron los materiales de fabricación, incorporando hueso, asta y marfil, con los que comenzaron a elaborarse agujas, puntas de flecha, punzones y buriles (Nowell, 2010). En definitiva, el repertorio tecnológico experimentó en un corto espacio de tiempo un gran incremento tanto en su complejidad funcional como en su variabilidad espacio-temporal.

Pero el cambio cultural que experimentaron las poblaciones homininas de hace 45.000 años no se limitó al repertorio de herramientas. A partir de este momento se hizo patente también la consolidación del uso del fuego para el procesamiento y la conservación de los alimentos, la explotación de los entornos costeros, la navegación marítima, el transporte de materias primas y útiles desde largas distancias (sugiriendo un incipiente comercio), la construcción de refugios como cabañas o chozas, y el incremento de las densidades de población. Comenzó la difusión del arte y de las representaciones figurativas, generalizándose el uso de la ornamentación y la decoración del cuerpo mediante el empleo de pigmentos, tatuajes y abalorios fabricados con conchas, dientes y huesos. Aparecieron además las primeras manifestaciones inequívocas del tratamiento ceremonial de la muerte y de comportamiento simbólico, junto con los primeros instrumentos musicales (Klein, 2009; Nowell, 2010).

Algunos (Klein, 2001; Mellars, 2005, 2007) ven en este cambio una auténtica revolución, una transformación profunda, súbita e imprevista. Otros investigadores, sin embargo, consideran que este modelo rupturista no se ajusta a la evidencia arqueológica y optan por un modelo de transición más gradual. Para Mcbrearty et al. (2000) el supuesto carácter abrupto de la transición a EPS/PS no es más que el resultado de una visión sesgada del registro arqueológico, en la medida en que el modo en que tuvo lugar esta transición en Europa (donde la introducción de estos rasgos sí tuvo lugar de manera abrupta) es tomado como modelo a nivel general, lo que no se justifica a la luz de los nuevos descubrimientos fuera de Europa, principalmente en África. El registro arqueológico africano muestra en época muy temprana elementos que anticipan lo que posteriormente definirá al repertorio cultural de la EPS/PS (Straus, 2009; Zilhão, 2007; d'Errico et al., 2011; Mcbrearty et al., 2000). Yacimientos como los de Stillbay y Howieson's Poort en Sudáfrica, con una antiguedad aproximada de 75.000 y 60.000 años respectivamente, o el de Kapthurin, en Kenia, con más de 230.000, contienen herramientas líticas con una morfología muy similar a las de la tecnología EPS/PS. Otros presentan herramientas fabricadas en hueso, como los de Blombos Cave, en Sudáfrica (aprox. 72.000 años) o Katanda, en República Democrática del Congo (más de 82.000 años); también abalorios realizados con conchas y muestras del uso 
de pigmentos en el yacimiento marroquí de Grotte de Pidgeons (aprox. 82.000 años); o restos de construcciones, como chozas, en Mumbwa Caves, Zambia, (aprox. 125.000 años).

En definitiva, los recientes descubrimientos arqueológicos parecen apuntar a que, aunque es en este momento en el que se difunden y consolidan estos rasgos, su origen es muy anterior. La transformación cultural que experimentaron las poblaciones humanas puede entenderse entonces como una reintroducción, una consolidación de ciertos rasgos ya presentes en algunas poblaciones de EPM/PM que acabaron por desaparecer sin conseguir difundirse. El carácter aparentemente revolucionario de este cambio vendría dado entonces por su rápida difusión y consolidación, no por una aparición súbita y monolítica, de tal manera que la transición entre los períodos EPM/ PM y EPS/PS poseería un mayor grado de continuidad de lo que se había considerado anteriormente (Straus, 2009).

Pero esto representa un problema. Si aceptamos las fechas de datación para los yacimientos africanos que contienen elementos característicos de EPS/ PS mucho antes de 45.000 años atrás, hemos de entender que la capacidades cognitivas necesarias para operar con la complejidad característica del comportamiento moderno estaban ya presente en estas poblaciones (d'Errico et al., 2011) aún cuando debido a ciertos factores estos avances no llegaran a difundirse y consolidarse. Las fechas de estos yacimientos apuntan incluso a la posibilidad de que dichos comportamientos hubieran estado asociados a nuestra especie desde su aparición (Mellard, 2005), e incluso algunos yacimientos europeos muestran elementos del tipo PS posiblemente asociados a otras especies como H. neanderthalensis (Zilhão, 2007; Foley et al., 1997). Sin embargo, por algún motivo la mayoría de estos rasgos culturales habrían acabado desapareciendo y reintroduciéndose a lo largo de toda la EPM/PM para terminar consolidándose y difundiéndose en torno a 45.000 años atrás. Si esto fue así, el principal problema que se nos presenta consiste en responder a la pregunta de qué factor, o factores, explican dicha consolidación.

\section{EXPLICACIONES PARA LA MODERNIDAD CONDUCTUAL}

Independientemente del momento en el que surgieran por primera vez los rasgos característicos del comportamiento moderno, lo cierto es que no fue hasta el comienzo de EPS/PS que no se hubieron consolidado entre casi todas las poblaciones humanas. Con lo cual, aun aceptando que dicha consolidación no fuera el resultado de un cambio abrupto y puntual, hemos de aceptar la existencia de un distanciamiento temporal de alrededor de 150.000 años entre la aparición de nuestra especie en el registro fósil y la difusión y predominio de estos caracteres modernos en la mayoría de las poblaciones humanas. El registro arqueológico muestra un marcado contraste para esas fechas, con una 
presencia de estos artefactos abundante y generalizada frente a una relativa escasez o total ausencia previa de los mismos en la mayoría de yacimientos. Por lo tanto, aunque la aparición de estos rasgos no tuviera lugar en este momento, debió existir alguna razón por la que las poblaciones humanas adoptaron en muy corto espacio de tiempo un estilo de vida tan marcadamente distinto al de los anteriores 150.000 años. Como aclararemos a continuación, las diferentes explicaciones que se han propuesto para identificar las causas de este acontecimiento pueden ser divididas en dos categorías, dependiendo de si consideran la emergencia del comportamiento moderno como resultado de un proceso a nivel individual o de un proceso a nivel colectivo.

\section{II.1. LA MODERNIDAD CONDUCTUAL COMO RESULTADO DE FACTORES ECONÓMICOS Y GENÉTICO-COGNITIVOS}

Algunos autores proponen que el factor que explica este escenario ha de buscarse en la transformación de ciertas capacidades cognitivas humanas como resultado de cambios genéticos que afectaron a la estructura cerebral (Klein, 2001; Coolidge et al., 2001). Tales explicaciones incluyen la capacidad para el pensamiento simbólico y abstracto, el carácter plenamente sintáctico del lenguaje, la autorreflexividad, una mayor memoria de trabajo junto con una mayor capacidad de previsión, planificación y atención, o una reorganización a gran escala en la interconexión de los distintos módulos mentales o inteligencias particulares (Knight et al., 1995; Nowell, 2010; Coolidge et al., 2001; Mithen, 1996).

Klein $(2001,2009)$ se ha constituido en el más firme defensor de la hipótesis de un cambio en la arquitectura cerebral como causa de la transformación cultural. Según él, la explicación que mejor se ajusta a las características del registro fósil es la de una mutación genética espontánea que dotara a nuestra especie de las habilidades cognitivas necesarias para producir el comportamiento moderno. Apoyándose en un estricto criterio de parsimonia, defiende dicha explicación en tanto que basada en un proceso al que se le atribuyen frecuentemente otros episodios de cambio conductual en nuestro linaje, como la aparición de las industrias olduvayense o achelense, asociadas a cambios en el tamaño cerebral o en la organización neurológica (Klein, 2009) sin necesidad de introducir nuevos mecanismos de cambio. A la luz de los estudios paleogenéticos más recientes, Klein ha rebajado su propuesta inicial de que una simple mutación fuese la causante de la reestructuración cerebral, pasando a considerar la posibilidad de que ésta se debiera a una combinación novedosa de los genes ya presentes en nuestro linaje, al no haberse encontrado en estos estudios ningún rastro de la supuesta fijación en el genoma de algún gen novedoso (Klein, 2019). El mayor obstáculo al que se enfrenta la propuesta de Klein, sin embargo, es la posibilidad de que, como 
precisamente parecen apuntar estos estudios paleogenéticos, las poblaciones humanas en África hubieran divergido con mucha anterioridad a las fechas en que supuestamente tuvo lugar esta reestructuración cerebral. Si esto es así, el supuesto cambio genético debería haber tenido lugar de manera independiente en cada una de las distintas poblaciones africanas, haberse producido una colonización por parte de una de estas poblaciones sobre el resto, o bien estar ausente en alguna de las poblaciones actuales, posibilidades todas sin apoyo en los estudios genéticos (Foley, 2006; Powell et al. 2010).

\section{II.2. LA MODERNIDAD CONDUCTUAL COMO RESULTADO DE FACTORES DEMOGRÁFICOS}

Recientemente, sin embargo, se ha venido considerando la posibilidad de que la consolidación de los rasgos conductuales modernos haya tenido lugar como consecuencia de ciertos cambios en las características sociales y demográficas de los grupos humanos, como variaciones en el tamaño de la población o la aparición de nuevas redes de interconexión entre sus miembros. En este sentido, la presencia intermitente del comportamiento moderno durante la EPM indicaría que la capacidad neurológica para el mismo estaba ya presente desde muy atrás (d'Errico et al., 2011) pero la ausencia de ciertas condiciones demográficas impidió su consolidación. Para los defensores de este enfoque, el nivel de desarrollo cultural que puede alcanzar y mantener en el tiempo una población está estrechamente ligado a sus características demográficas, de tal forma que tanto la aparición como la pérdida de rasgos culturales complejos vienen a ser el resultado de alteraciones que se producen a escala social (Sterelny, 2020).

Desde principios del siglo XXI ha venido cobrando fuerza dentro de los estudios arqueológicos lo que Collard et al. (2016) denominan como un giro demográfico, una proliferación de modelos que se apoyan en factores sociales (principalmente el aumento o la disminución del tamaño de la población) para explicar los grandes cambios en el registro arqueológico. El origen de la controversia actual en torno a estos modelos hay que situarlo principalmente en el estudio de Shennan (2001), quien muestra que las consecuencias de las innovaciones culturales afectan en mayor o menor medida a una población dependiendo fundamentalmente de su tamaño. El modelo presentado por Shennan muestra que cuando la transmisión cultural dentro de una población tiene lugar de manera vertical (entre miembros de una generación $\mathrm{y}$ sus descendientes directos) u oblicua (entre miembros de una generación e individuos no emparentados de la siguiente), en las poblaciones que son pequeñas es más probable que pervivan variantes culturales perniciosas por un proceso análogo al de la deriva genética. En una población pequeña la variabilidad cultural será normalmente más baja que en poblaciones grandes, 
y esta baja variabilidad puede ocasionar que, por azar, la variante cultural más común sea una variante perniciosa. Al aumentar el tamaño de la población aumenta también la probabilidad de que las variantes beneficiosas amplíen su proporción con respecto a las perjudiciales, ya que la ventaja que proporcionan a sus portadores está menos expuesta a perderse por acontecimientos azarosos, con lo que habrá una mayor probabilidad de que estos individuos sean tomados como modelos a imitar (Shennan, 2001, p. 12). En el caso de la transición de EPM a EPS, Shennan atribuye la consolidación del comportamiento moderno a la mejora de las condiciones climáticas producida en torno a 60.000 años atrás, que trajo consigo un aumento en el tamaño de las poblaciones humanas tras haber superado un cuello de botella demográfico de unos 10.000 años de duración, ${ }^{3}$ y no a un incremento súbito de las capacidades cognitivas individuales. A medida que las condiciones climáticas se iban haciendo más favorables y los grupos humanos iban creciendo en tamaño, las innovaciones culturales que los individuos, con idénticas capacidades cognitivas que sus predecesores, iban introduciendo podían ser conservadas y acumuladas. Este incremento cultural permitió mantener un mayor aprovechamiento de los recursos y potenciar más aún el incremento demográfico, lo que se tradujo a su vez en una rápida aceleración del desarrollo cultural.

En el modelo de Shennan, la característica demográfica que posibilita la permanencia de las innovaciones beneficiosas es el tamaño: a mayor número de individuos conformando una población, mayor probabilidad de que ésta, en su conjunto, retenga dichas innovaciones. Hemos de aclarar, sin embargo, que la noción de tamaño de población a la que se refiere Shennan, así como la que suele emplearse en la mayoría de los modelos demográficos, es en realidad una noción más restrictiva que la de tamaño absoluto o total de la población. Al referirse al tamaño de una población aluden a su tamaño efectivo, que no tiene por qué ser igual al tamaño absoluto. Lo que cuenta a la hora de estudiar la influencia de los factores demográficos en la transmisión cultural es el subconjunto de individuos potencialmente involucrados en el proceso de transmisión cultural, medida que referirá a un subconjunto diferente en función de las necesidades del modelo con el que se estudie la dinámica de esta transmisión, como el número de maestros o de aprendices potenciales.

$\mathrm{Al}$ aumentar el tamaño absoluto de la población es probable que también lo haga su tamaño efectivo, aunque no necesariamente, pues la probabilidad de interacción entre maestros y aprendices no depende exclusivamente de su

3 Klein (2009) aduce precisamente esta situación como una prueba en contra de quienes defienden la influencia de los factores demográficos en la transición EPM/EPS, ya que antes de que tuviera lugar dicha transición el tamaño poblacional medio en África había disminuido considerablemente. 
número sino también de la manera en que interactúan (Kobayashi et al., 2012; Henrich, 2004). Tanto el número de miembros como el grado de interconexión contribuyen al valor del tamaño efectivo de la población: cuanto mayor sea la interconexión entre ellos, mayor será el número de maestros o aprendices potenciales para otros individuos. Una población más pequeña que otra en términos absolutos puede tener un mayor tamaño efectivo de población si el número de quienes pueden participar en el proceso de transmisión cultural es mayor, debido a que su estructura social favorece dicha participación. De la misma manera, si una población aumenta su tamaño pero reduce la conectividad de sus miembros puede disminuir su tamaño efectivo.

\section{CONEXIÓN ENTRE DEMOGRAFÍA Y COMPLEJIDAD TECNOLÓGICA}

A partir del estudio de Shennan se han propuesto toda una serie de modelos que examinan la conexión entre este tamaño efectivo y distintas dimensiones o características del desarrollo cultural, siendo mayoritario el número de modelos que lo relacionan con alguna medida de la complejidad tecnológica, entendida normalmente como proxy o indicador del grado de complejidad cultural general de una población (Fogarty et al., 2016). Sterelny (2020) distingue dos medidas diferentes de complejidad tecnológica comúnmente utilizadas dentro de estos modelos. Por un lado, puede aparecer representada como una medida del tamaño del repertorio o número de utensilios diferentes empleados por una comunidad o grupo. En este sentido, para determinar cuán complejo sea este repertorio habría de tenerse en cuenta únicamente su tipología, no las características de los utensilios en sí mismos. Debería decirse entonces, de una población que contara con un mayor número de herramientas diferentes que otra, que su complejidad tecnológica es mayor aun cuando dichas herramientas difiriesen en su estructura interna.

Una segunda medida es la de la complejidad máxima, entendida como el número de elementos tecnológicos funcionalmente diferenciados que componen los utensilios de mayor complejidad del repertorio (Sterelny, 2020). Estos elementos vienen a constituir distintas tecnounidades (Oswalt, 1987), de tal forma que dos herramientas compuestas por el mismo número de tecnounidades poseerían el mismo grado de complejidad, con independencia de que difieran en otras características como el material del que están fabricadas o el tiempo de elaboración. La medida de la complejidad en tanto que número de tecnounidades se vuelve sin embargo problemática cuando se intenta comparar utensilios en los que, aun contando con el mismo número de partes funcionales, el grado de sofisticación de estas partes consideradas de forma aislada sea muy diferente en unos y otros. De la misma manera, utensilios pertenecientes a un mismo linaje cultural, resultado de una transformación sucesiva desde formas más arcaicas, pero a los que no se les hubiera añadido ni 
eliminado ninguno de sus elementos funcionales, habrían de ser considerados como pertenecientes a un mismo grado de complejidad tecnológica. Por este motivo otros autores prefieren una definición de complejidad que incida en las características de la relación entre estos elementos funcionales más que en su número, como la establecida con respecto al grado de interacción e interdependencia entre componentes (Querbes et al., 2014). Sin embargo, a pesar de estas dificultades, la noción de complejidad máxima sigue siendo muy utilizada en los estudios demográficos, posiblemente debido a que la sencillez de su carácter aditivo la hace más operativa en la construcción de modelos frente a nociones que se fundamentan en aspectos más difícilmente cuantificables.

\section{III.1. INCREMENTO DE LA COMPLEJIDAD TECNOLÓGICA DEBIDO A LA PRESIÓN DEMOGRÁFICA}

Independientemente del sentido en que se entienda el concepto de complejidad tecnológica, podemos distinguir dos maneras en que los modelos que la conectan con las características demográficas abordan la naturaleza de esta relación (Andersson et al., 2016; Shennan, 2015).

La primera consiste en relacionar el aumento en la densidad demográfica con la necesidad de encontrar nuevas soluciones a problemas relacionados con el incremento de la presión sobre los recursos o a necesidades en la organización social. Cuando una población aumenta su densidad de población aumenta la probabilidad de que aparezcan innovaciones culturales debido a que éstas se hacen necesarias para satisfacer nuevas necesidades. Por ejemplo, Kuhn y Stiner (2007) relacionan la aparición de elementos ornamentales con el aumento de las interacciones humanas como consecuencia del aumento en la densidad demográfica. Debido al rápido crecimiento demográfico posterior al cuello de botella por el que pasaron las poblaciones paleolíticas, las interacciones sociales hubieron de hacerse más complejas en tanto que aumentaba el número de encuentros por individuo a lo largo del día. De esta manera se reforzaría la necesidad de transmitir la propia identidad, la jerarquía o la pertenencia a un grupo cultural diferenciado, emergiendo así la ornamentación corporal como una nueva manera de comunicación. Según estos autores, la aparición de nuevas técnicas de subsistencia y el cambio en la dieta hacia el consumo de animales más pequeños (de bajo valor nutricional y difícil obtención) tuvo lugar del mismo modo, por la necesidad de extraer más recursos de una misma porción de territorio como consecuencia del aumento demográfico (Kuhn et al., 2001). El principal mecanismo por el que este aumento demográfico produce entonces la aparición de artefactos más complejos es el incremento en los niveles de competitividad, como consecuencia de la presión demográfica sobre los recursos (Read et al., 2003). 


\section{III.2. INCREMENTO DE LA COMPLEJIDAD TECNOLÓGICA DEBIDO A LA EFICACIA}

\section{EN EL PROCESO DE ACUMULACIÓN CULTURAL}

Una segunda manera de relacionar demografía y complejidad es a través de la incidencia del aumento demográfico en el proceso de acumulación cultural entre generaciones. El aumento de la población puede condicionar los procesos de transmisión cultural de tal forma que la capacidad de retención de las innovaciones se vea alterada, afectando al desarrollo del repertorio cultural a lo largo del tiempo. El nivel de complejidad cultural alcanzado por una población depende entonces del efecto limitador o impulsor de ciertos procesos de interacción social, con lo que la identificación de estos procesos y del alcance de sus efectos serán los propósitos de estos modelos demográficos (Andersson et al., 2016).

La idea central de estos modelos reside en la consideración del desarrollo cultural y tecnológico como un proceso acumulativo, en el que la retención de innovaciones a lo largo del tiempo produce normalmente un aumento paulatino en el nivel de complejidad (Shennan, 2015). Las sucesivas generaciones conservan las innovaciones beneficiosas dentro del repertorio cultural el tiempo suficiente como para que puedan ser mejoradas en algún momento posterior, por medio de algún mecanismo de retención generalmente relacionado con una capacidad de transmisión cultural más eficaz, lo cual puede llevarse a cabo de diferentes maneras:

i. Aumentando la eficacia al evitar que se pierdan los individuos que poseen las variantes.

Un primer modo en que puede incrementarse esta eficacia consiste en reducir la posibilidad de que una variante desaparezca con los individuos que la poseen, aumentando así indirectamente la probabilidad de que la variante sea imitada. De esta manera, las poblaciones grandes se protegen de la pérdida de las innovaciones simplemente porque en cada generación existe un número suficiente de individuos que pueden transmitir la variante cultural. Sterelny (2020) distingue dos maneras en que puede tener lugar de este modo la retención de información cultural entre generaciones: por redundancia (al haber más individuos poseedores de información se reduce la posibilidad de que ésta se pierda) y por especialización (cuando los individuos pueden dedicar más tiempo a una tarea aumentan su nivel de habilidad para esa tarea y se incrementa así la probabilidad de innovación).

ii. Aumentando la eficacia al incrementar el número de transmisiones.

Cuando las poblaciones son pequeñas es más probable que las innovaciones beneficiosas desaparezcan debido al mayor impacto relativo de los errores en la transmisión de dichas innovaciones entre individuos. En las poblaciones 
grandes, estos errores se ven compensados por el hecho de que la transmisión tiene lugar un mayor número de veces, con lo que la probabilidad de que el error acabe eliminando una innovación cultural en la población es menor. De esta forma, a medida que una población va creciendo aumenta la probabilidad de que las innovaciones introducidas sean retenidas.

iii. Aumentando la eficacia al aumentar la habilidad media de los individuos.

Otra manera consiste en reducir los efectos perniciosos de las imprecisiones en la transmisión cultural, contrarrestados por un mayor nivel de habilidad de los individuos que participan en dicha transmisión (Henrich, 2004; Powell et al., 2009). Según estos modelos, para retener el nivel de complejidad cultural existente es necesario que el nivel de habilidad medio de los individuos se mantenga, pues en caso contrario la población no podría hacer frente a los errores en la transmisión y el nivel de complejidad disminuiría. La clave para entender cómo estos modelos conectan el tamaño demográfico con la complejidad cultural va a estar entonces en el mecanismo que propongan para explicar cómo este tamaño incide en el nivel de habilidad medio, y cómo esto se traduce en un incremento o disminución del grado de complejidad cultural.

Henrich (2004) explora este mecanismo en el caso muy debatido de la aparente pérdida de complejidad cultural en Tasmania durante el Holoceno. Al finalizar la última época glacial hace unos 12.000 años y con la consiguiente subida del nivel del mar, los habitantes de Tasmania quedaron desconectados de las poblaciones de Australia y su número disminuyó considerablemente (Jones 1995). A partir de ese momento comenzó una progresiva reducción de la complejidad del repertorio tecnológico que en algunos casos supuso la total desaparición de elementos que llevaban presentes desde la ocupación de Tasmania unos 22.000 años antes, tales como las herramientas de hueso, el utillaje de pesca, los propulsores, los arcos y las flechas, la producción intencionada del fuego, la fabricación de canoas o las vestimentas de piel, elementos todos, como hemos apuntado, característicos de la modernidad anatómica de nuestra especie. ${ }^{4}$ No en vano, la cultura tasmana llegó a ser considerada como la cultura material más simple de las sociedades modernas (Diamond, 1978). Así, mientras que las poblaciones de la Australia continental poseían un repertorio tecnológico compuesto por cientos de útiles diferentes, según Henrich (2004) el utillaje tasmano en la época en que entraron en contacto con los europeos se componía tan sólo de unos veinticuatro, aunque algunos autores ponen en duda esta afirmación (cf. Collard et al., 2016).

Henrich (2004) establece una relación causal entre el aislamiento sufrido por la sociedad tasmana y la pérdida de complejidad tecnológica sobre la

4 Sollas (1911) llegó a comparar la tecnología tasmana con el grado de desarrollo de la cultura musteriense (cf. Haidle, 2016). 
base de que su tamaño demográfico era insuficiente para mantener por sí misma el nivel de complejidad exhibido durante el período de contacto con las poblaciones de la Australia continental. Para mostrar esto elabora un modelo según el cual los individuos de una población $N$ (conjunto efectivo de interactuantes en el proceso de transmisión) tratan de aprender una habilidad imitando al individuo más hábil o cualificado de la población, asumiendo que siempre son capaces de identificarlo. El nivel de habilidad es representado por $z$, siendo $z_{h}$ el nivel de habilidad del individuo más hábil $(h)$, de tal forma que cada individuo de $N$ que aprende de $h$ acaba con un valor de $z$ tal que algunos superan $z_{h}$ y otros quedan por debajo.

Toda transmisión está sujeta a un cierto grado de imperfección debido a los errores que se producen durante en el aprendizaje, siendo así que cuanto mayor sea el nivel de complejidad de una habilidad (representado por $\alpha$ ), mayor será la probabilidad de que se produzcan errores durante la transmisión. Así, cuando el valor de $\alpha$ es alto, la mayor parte de los individuos fallarán en el intento de adquirir la habilidad y su valor de $z$ será bajo, con lo que la diferencia entre el valor medio de $z$ para la población y $z_{h}$ será grande.

Sin embargo, en cada generación existe una cierta probabilidad de que un miembro $h$ ' incremente su valor individual de $z$ de tal forma que supere el máximo actual. De esta manera se incrementaría el valor máximo de $z$ (ahora $z_{h}$ ), aumentando también la diferencia existente entre los valores medio y máximo de $z$ para la población. Cuando aumenta el tamaño de la población es más probable que un individuo supere $z_{h}$ y que haya otros que puedan igualarle, con lo que la habilidad compleja puede mantenerse en la población y pueden también aparecer otras más complejas. El valor esperado de $z_{h}$ es, por lo tanto, mayor al aumentar la población.

Por contra, cuando disminuye el tamaño de la población se reduce el número de habilidades complejas (con un valor alto de $\alpha$ ) presentes en ella, ya que la probabilidad de que alguien supere o iguale $z_{h}$ en esa generación es menor. En la siguiente generación, si ningún individuo ha alcanzado $z_{h}$ el nuevo valor máximo será inferior, y así sucesivamente para las demás generaciones hasta que el nivel de $z_{h}$ se reajuste con la probabilidad de que haya individuos que lo alcancen, asociada al tamaño de la población. En definitiva, puesto que el valor de $z_{h}$ va disminuyendo, las habilidades más complejas no pueden ser mantenidas y van desapareciendo del repertorio.

El valor de $\alpha$ está por lo tanto relacionado con un determinado valor de $z_{h}$ que dependerá del tamaño de $N$ en cada generación, lo que significa que para que se mantenga el nivel de complejidad es necesario que el tamaño de la población supere un determinado umbral, por debajo del cual los valores altos de $\alpha$ empezarán a desaparecer como consecuencia de la disminución en $z_{h}$ asociada a la reducción de $N$. Si el tamaño de la población se mantiene 
por encima de ese umbral, las habilidades con un alto valor de $\alpha$ podrán mantenerse o aumentar en número. Lo cual significa que la presencia de elementos complejos dependerá, en último término, del tamaño efectivo de esa población.

Henrich concibe la simplificación del repertorio tecnológico tasmano como resultado de esta reducción en el valor de $z$ necesario para sostener las habilidades complejas. Al quedar incomunicados tras la subida del mar, el tamaño de $N$ se redujo (recordemos que el tamaño que interesa es el tamaño efectivo, para el cual contaban también los individuos del continente australiano en tanto que potencialmente involucrados en el proceso de transmisión), con lo que la existencia de un nivel de habilidad medio para fabricar útiles complejos como, por ejemplo, redes de pesca, también disminuyó. Su población no era lo suficientemente grande como para superar el umbral de $N$ necesario para mantener el nivel de complejidad de unos utensilios que, poco a poco, fueron perdiendo, hasta acabar con un utillaje tecnológico muy reducido y compuesto de elementos muy simples. ${ }^{5}$

Este modelo gozó de una gran popularidad en los estudios etnográficos y arqueológicos, y contribuyó a la aceptación de las explicaciones del cambio cultural basadas en factores demográficos (Kline et al., 2010; Kempe et al., 2014; Richerson, 2013). Sin embargo, esta aceptación ha sido llevada a cabo muchas veces con demasiado optimismo, sin tener en cuenta las dificultades que suelen presentarse para descartar explicaciones alternativas (Fogarty et al., 2016). Varios estudios apuntan a una mejor correlación entre complejidad tecnológica y factores de tipo no demográfico, como la variabilidad ambiental o el riesgo de escasez de los recursos. Collard et al. (2005) comparan la eficacia del tamaño demográfico como factor explicativo con los factores de movilidad, tipo de dieta y riesgo de escasez de recursos para otras sociedades de cazadores-recolectores sin encontrar ninguna correlación entre el primero y la complejidad del utillaje tecnológico. Tan sólo el riesgo de escasez de recursos se revela en su análisis como predictor de la complejidad tecnológica.

Read (2006) considera que el nivel de complejidad de la tecnología tasmana se ajusta a lo que debería esperarse para las condiciones climáticas y las características de los recursos disponibles en el entorno. Según Read, la naturaleza y distribución de estos recursos no precisaba para su explotación de una tecnología más compleja que la del reducido utillaje de los tasmanos, por lo que la desaparición de los útiles complejos se explicaría mejor por causas de tipo económico y no demográfico. Como resultado de una valoración entre

5 Esta dificultad de una población para mantener un sistema tecnológico y cultural complejo cuando disminuye su tamaño ha sido denominada a raíz de la propuesta de Henrich como «efecto tasmano» (Richerson et al., 2009). 
los costes y beneficios de mantener una tecnología más costosa desde el punto de vista del esfuerzo y del tiempo de aprendizaje, en relación a unos resultados sólo ligeramente mayores, los tasmanos habrían acabado por desecharlas, conservando sólo aquellas que produjeran los beneficios necesarios con un menor esfuerzo. Henrich (2006) contradijo esta interpretación en base a la falta de evidencias de que los tasmanos realizaran de hecho una estimación entre costes y beneficios. En tal caso, quienes realizaran esta estimación habrían primero de experimentar individualmente con la fabricación de estos utensilios y valorar después la eficiencia de los mismos, de modo que el registro arqueológico habría de contener numerosos elementos inacabados o inservibles, lo cual no ocurre. De igual manera, según Henrich, dicha interpretación niega la posibilidad de que se produzca un cambio cultural mientras la relación entre costes y beneficios se mantenga en el tiempo, por lo que en un entorno en el que esta relación se mantuviera inalterada el repertorio cultural de las sociedades que lo habitan no habría de sufrir modificaciones una vez alcanzado una relación óptima, lo cual sería, cuanto menos, discutible (Henrich, 2006).

Vaesen et al. (2016) y Read (2011) critican el modelo de Henrich aduciendo que parte de unos supuestos implausibles. Dicho modelo no sería de utilidad en la explicación del cambio tecnológico para estas sociedades en tanto que la manera en que los individuos suelen aprender en la vida real no se ajusta a esta pauta de imitar al individuo con mayor nivel de habilidad, sino que generalmente imitan a los progenitores o a otros individuos de nivel más bajo que sean accesibles. Por lo tanto, dado que el supuesto inicial no se cumple, sus predicciones no tendrían valor explicativo sobre el problema que intenta resolver. Henrich (2006; Henrich et al., 2016) ha tratado de justificar la elección de este modo de aprendizaje en tanto que la presencia de transmisión vertical no altera los resultados del modelo (véase Henrich, 2004, p. 205). En esta línea, Powell et al. (2009) extienden el modelo de Henrich para incorporar una cierta cantidad de transmisión vertical en el aprendizaje, ajustando a su vez las condiciones demográficas iniciales para equipararlas a las características de las poblaciones europeas durante el tránsito del PM/PS. Aplicando una regla de aprendizaje basada en aprender primero de uno de los progenitores y comparar después con otro individuo de la población, analizan cómo evoluciona el nivel de una habilidad en base al grado de interconexión demográfico de una metapoblación compuesta por varias subpoblaciones partiendo de los valores de densidad poblacional estimada para las poblaciones del Pleistoceno. Cuando dicha metapoblación se estructura de tal manera que aumenta el flujo de información (o de individuos) entre las distintas subpoblaciones, el resultado obtenido cuando los individuos aplican esta regla de aprendizaje es un incremento en la capacidad de dicha metapoblación 
para retener las innovaciones culturales. Concluyen así que tras la mejora de las condiciones climáticas durante el Pleistoceno y el consiguiente aumento demográfico se habría producido en Europa una situación inversa al «efecto tasmano», aumentando el tamaño efectivo al incrementarse los contactos y las transferencias de información entre los diferentes subgrupos que habían permanecido aislados. Tal situación se habría repetido numerosas veces, aunque a menor escala, probablemente desde la aparición de $H$. sapiens anatómicamente moderno, cuando se produjeron en diferentes regiones de África picos de densidad demográfica local de corta duración que permitieron alcanzar un tamaño efectivo de población suficiente para producir y mantener elementos de tipo moderno. Al existir una correlación entre las estimaciones de densidad demográfica y los tiempos de aparición del comportamiento moderno en Europa y África, concluyen que el factor que mejor predice de manera general el incremento en la complejidad cultural es el demográfico, aunque los resultados de su modelo no se ajustan al patrón de aparición del comportamiento moderno en Asia (Collard et al., 2016).

\section{CONCLUSIONES}

La insistencia en la búsqueda de factores genético-cognitivos para explicar el surgimiento del comportamiento humano moderno, culturalmente complejo y diverso, choca con la evidencia etnográfica actual que revela la existencia de sociedades con una tecnología relativamente simple y cercana en muchos aspectos a las características de la tecnología paleolítica. Salvo que apelemos a una supuesta diferencia cognitiva heredable entre los individuos de distintas sociedades actuales, algo que los estudios genéticos parecen descartar, habremos de aceptar la existencia de otros factores que expliquen las diferencias en el grado de complejidad tecnológica y cultural de estas mismas sociedades.

Cada sociedad humana actual ha seguido su propio desarrollo histórico. A pesar de las posibles similitudes que puedan existir entre las sociedades de cazadores-recolectores paleolíticas y las actuales, cada grupo humano ha debido lidiar con unas condiciones ecológicas particulares y encontrar soluciones que les permitieran afrontarlas, por lo que no podemos estar seguros de que ninguna de las que existen hoy en día pueda utilizarse como modelo para estudiar las sociedades paleolíticas (Cohen, 1985). Pero el hecho de que en las sociedades actuales existan estas diferencias y que éstas no sean atribuibles fácilmente a factores genético-cognitivos puede arrojar luz sobre lo ocurrido en el paso de EPM/PM a EPS/PS en el caso de que, como apuntan los descubrimientos actuales, la emergencia del comportamiento moderno no tuviera un carácter revolucionario sino que más bien su consolidación se 
debiera a un cambio en la distribución de frecuencias de unos rasgos que ya estaban presentes mucho tiempo atrás.

Distintas propuestas se han planteado para responder a la pregunta de qué factor explica dicha consolidación, de entre las cuales ha cobrado una excepcional popularidad la que considera que el grado de desarrollo tecnológico de una población está estrechamente relacionado con sus características demográficas. Teniendo en cuenta esta afirmación, la modernidad conductual no habría de considerarse entonces como una propiedad a nivel individual, sino colectiva. La aplicación de estos modelos al campo de la paleoantropología y la etnografía dio lugar a un optimismo y una confianza en los factores demográficos como predictores del cambio cultural que ha ido rebajándose recientemente (Sterelny, 2020). El modelo de Henrich (2004) abrió la posibilidad de que el análisis de la tecnología desde planteamientos demográficos para las sociedades actuales revelase la conexión entre el grado de complejidad tecnológica alcanzado y su tamaño efectivo. De esta forma, el estudio de la aparente degradación cultural de Tasmania apuntaría a un escenario para las poblaciones PM/PS en que éstas hubieran sufrido una situación inversa a la del caso tasmano, siendo la consolidación de la modernidad conductual el resultado de un aumento en el tamaño o en la conectividad de las poblaciones humanas durante el Pleistoceno (Powell et al., 2009).

Pero la aplicación de estos modelos demográficos al análisis tecnológico ha de tener en cuenta una serie de dificultades que hacen necesario reconsiderar las expectativas iniciales. En primer lugar, las fuentes etnográficas y arqueológicas difieren tanto en el concepto de complejidad empleado como en los resultados cuantitativos ofrecidos para establecer el grado de complejidad tecnológica de una sociedad, lo cual hace difícil contrastar las predicciones de los modelos que la abordan. La falta de un criterio unitario para cuantificar el grado de complejidad del utillaje tecnológico permite interpretar y reajustar los datos de tal manera que modelos contrapuestos pueden reclamar su mejor encaje con estos datos. ${ }^{6}$ Segundo, en tanto que modelos idealizados, los modelos demográficos no tienen en cuenta la distribución real de las diferentes poblaciones humanas en un entorno ecológico que pocas veces reduce por completo la conexión entre ellas. De esta manera la interpretación del «efecto tasmano» no tiene en cuenta las posibilidades de contacto y transmisión cultural llegada desde poblaciones que no han sufrido aislamiento. ${ }^{7}$ En tercer

6 Véase la disputa entre Read (2006) y Henrich (2006).

7 En el caso de Tasmania, el modelo de Henrich no tiene en cuenta que podría haberse producido difusión de utensilios complejos por individuos provenientes de la Australia continental, quienes sí poseían la tecnología suficiente para cruzar el estrecho de Bass (Sterelny, 2020). 
lugar, estos modelos dejan sin considerar un aspecto clave de la noción misma de artefacto tecnológico, a saber, que toda tecnología guarda relación con la tarea para la que se elabora. La noción puramente abstracta de cantidad de herramientas o de número de partes constituyentes informa muy poco acerca de la utilidad de los artefactos tecnológicos y de su eficacia. Desde un punto de vista instrumental (aunque no desde el ornamental o simbólico), un simple trozo de madera puede ser lo suficientemente eficaz (para usarse, por ejemplo, de bastón) como para no complicar innecesariamente su estructura añadiendo más elementos. Considerar cómo afectan exclusivamente las características demográficas al incremento o disminución de la complejidad tecnológica sin tener en cuenta otras dimensiones de los artefactos tecnológicos más allá de la cuantificación de sus partes o su variedad difícilmente puede dar una respuesta satisfactoria a la pregunta de qué propició la consolidación de una transformación tan decisiva y ubicua como la experimentada por las poblaciones humanas del Pleistoceno.

La mayoría de los modelos utilizados para explicar la transición de PM/ PS han tratado de encontrar el factor, el único o más decisivo determinante que provocara el paso hacia lo que nos ha traído hasta el lugar que ocupamos hoy en día como especie. En este artículo se han presentado estos modelos dentro del marco general de los intentos por identificar dicho factor, aportando una clasificación que facilite la comprensión del alcance y del papel de las propuestas basadas en factores demográficos. A su vez, se ha mostrado cómo los modelos demográficos han de tomarse con cautela a pesar de su popularidad, resultando problemáticos cuando se emplean de manera aislada. El enfoque necesario para explicar la dinámica tecnológica y cultural humana ha de partir por el contrario de una integración entre factores con un alcance heterogéneo, lo cual no es sencillo. Para lograr esto los modelos demográficos siguen siendo una herramienta esencial, y nuevos desarrollos que parten desde supuestos más elaborados (ej. Aoki, 2019; Kobayashi et al., 2016) están contribuyendo a esclarecer el alcance de estos factores, pero sigue siendo preciso considerar con cautela sus implicaciones, a riesgo de caer en simplificaciones excesivas.

\section{REFERENCIAS BIBLIOGRÁFICAS}

ANDERSSON, C. y READ, D., 2016. «The evolution of cultural complexity: Not by the treadmill alone». Current Anthropology, 57(3), 261-286. http://dx.doi. org $/ 10.1086 / 686317$

AOKI, K., 2019. «Cultural bistability and connectedness in a subdivided population». Theoretical Population Biology. 129, 103-117. https://doi.org/10.1016/j. tpb.2019.03.009 
COHEN, M., 1985. «Prehistoric hunter-gatherers: the meaning of social complexity». En T. D. Price y J. A. Brown, (eds.), Prehistoric hunter-gatherers: the emergence of cultural complexity. Waltham, MA: Academic Press, pp. 99-119.

COLLARD, M., KEMERY, M. y BANKS, S., 2005. «Causes of Toolkit Variation Among Hunter-Gatherers: A Test of Four Competing Hypotheses». Canadian Journal of Archaeology / Journal Canadien D'Archéologie, 29(1), 1-19.

COLLARD, M., VAESEN, K., COSGROVE, R. y ROEBROEKS, W., 2016. «The empirical case against the "demographic turn" in Palaeolithic archaeology». Philosophical Transactions of the Royal Society of London, B: Biological Sciences, 371, 20150242. https://doi.org/10.1098/rstb.2015.0242

COOLIDGE, F. y WYNN, T., 2001. «Executive Functions of the Frontal Lobes and the Evolutionary Ascendancy of Homo Sapiens». Cambridge Archaeological Journal. 11. https://doi.org/10.1017/S0959774301000142

D'ERRICO, F. y STRINGER, C. B., 2011. «Evolution, revolution or saltation scenario for the emergence of modern cultures?». Philosophical transactions of the Royal Society of London. Series B, Biological sciences, 366(1567), 1060-1069. https:// doi.org/10.1098/rstb.2010.0340

DIAMOND, J., 1978. "The Tasmanians: the longest isolation, the simplest technology». Nature, 273, 185-186.

FOGARTY, L., WAKANO, J., FELDMAN, M. y AOKI, K., 2016. «The Driving Forces of Cultural Complexity: Neanderthals, Modern Humans, and the Question of Population Size». Human Nature. 28. https://doi.org/10.1007/s12110-0169275-6

FOLEY, R., 2006. «The emergence of culture in the context of hominin evolutionary patterns». En S. Levinson y P. Jaisson (eds.), Evolution and Culture. Cambridge: MIT Press, pp. 53-77.

FOLEY, R. y LAHR, M., 1997. «Mode 3 Technologies and the Evolution of Modern Humans». Cambridge Archaeological Journal, 7(1), 3-36. https://doi. org/10.1017/S0959774300001451

HAIDLE, M. N., 2016. «Lessons from Tasmania - Cultural Performance Versus Cultural Capacity». En M. Haidle, N. Conard y M. Bolus (eds.), The Nature of Culture. Springer, Dordrecht, pp. 7-17. https://doi.org/10.1007/978-94-0177426-0_2

HENRICH, J., 2004. «Demography and cultural evolution: how adaptive cultural processes can produce maladaptive losses: the Tasmanian case». American Antiquity 69, 197-214. https://doi.org/10.2307/4128416

HENRICH, J., 2006. «Understanding cultural evolutionary models: a reply to Read's critique». American Antiquity, 71(4), 771-782. https://doi.org/10.2307/40035890

HENRICH, J., BOYD, R., DEREX, M., KLINE, M., MESOUDI, A., MUTHUKRISHNA, M., POWELL, A., SHENNAN, S. y THOMAS, M., 2016. «Understanding cumulative cultural evolution». Proceedings of the National Academy of Sciences Nov 2016, 113 (44), E6724-E6725. https://doi.org/10.1073/ pnas. 1610005113

JONES, R., 1995. «Tasmanian Archaeology: Establishing the Sequence». Annual Review of Anthropology, 24, 423-446 
KEMPE, M., y MESOUDI, A., 2014. «An experimental demonstration of the effect of group size on cultural accumulation». Evolution and Human Behavior, 35(4), 285-290. https://doi.org/10.1016/j.evolhumbehav.2014.02.009

KLEIN, R., 2001. «Southern Africa and Modern Human Origins». Journal of Anthropological Research, 57, 1-16. https://doi.org/10.1086/jar.57.1.3630795

KLEIN, R., 2009. The human career: human biological and cultural origins. Chicago: Chicago University Press.

KLEIN, R., 2019. «Population structure and the evolution of Homo sapiens in Africa». Evolutionary Anthropology: Issues, News, and Reviews, 28. https://doi. org/10.1002/evan.21788

KLINE, M. A. y BOYD, R., 2010. «Population size predicts technological complexity in Oceania». Proceedings of the Royal Society B: Biological Sciences, 277, 25592564. https://doi.org/10.1098/rspb.2010.0452

KNIGHT, C., POWER, C. y WATTS, I., 1995. «The human symbolic revolution: a Darwinian account». Cambridge Archaeological Journal, 5(1), 75-114. https:// doi.org/10.1017/S0959774300001190

KOBAYASHI, Y. y AOKI, K., 2012. «Innovativeness, population size and cumulative cultural evolution». Theoretical population biology, 82(1), 38-47. https://doi. org/10.1016/j.tpb.2012.04.001

KOBAYASHI, Y., OHTSUKI, H., y WAKANO, J. Y., 2016. «Population size vs. social connectedness - A gene-culture coevolutionary approach to cumulative cultural evolution». Theoretical population biology, 111, 87-95. https://doi. org/10.1016/j.tpb.2016.07.001

KUHN, S. y STINER, M., 2007. «Paleolithic ornaments: implications for cognition, demography and identity». Diogenes, 54, 40-48. https://doi. org/10.1177/0392192107076870

KUHN, S., STINER, M., REESE, D. S., y GÜLEÇ, E., 2001. «Ornaments of the earliest Upper Paleolithic: New insights from the Levant». Proceedings of the National Academy of Sciences of the United States of America, 98(13), 76417646. https://doi.org/10.1073/pnas.121590798

MCBREARTY, S., y BROOKS, A. S., 2000. «The revolution that wasn't: A new interpretation of the origin of modern human behavior». Journal of Human Evolution, 39, 453-563. https://doi.org/10.1006/jhev.2000.0435

MELLARS, P., 2005. «The impossible coincidence: A single-species model for the origins of modern human behavior in Europe». Evolutionary Anthropology, 14, 12-27. https://doi.org/10.1002/evan.20037

MELLARS P., 2007. «Rethinking the human revolution: Eurasian and African perspectives». En P. Mellars, K. Boyle, O. Bar-Yosef y C. Stringer (eds.), Rethinking the Human Revolution. Cambridge: MacDonald Institute, pp. 1-14.

MITHEN, S., 1996. The Prehistory of Mind: The Cognitive Origins of Art, Religion and Science. London: Thames \& Hudson.

NOWELL, A., 2010. «Defining Behavioral Modernity in the Context of Neandertal and Anatomically Modern Human Populations». Annual Review of Anthropology, 39, 437-52. https://doi.org/10.1146/annurev.anthro.012809.105113 
OFEK, H., 2001. Second Nature: Economic Origins of Human Evolution. Cambridge, Cambridge University Press.

OSWALT, W. H., 1987. «Technological complexity: the Polar Eskimos and the Tareumiut». Arctic Anthropology, 24, 82-98.

POWELL, A., SHENNAN, S. y THOMAS, M. G., 2009. «Late pleistocene demography and the appearance of modern human behavior». Science 324, 1298-1301. https://doi.org/10.1126/science.1170165

POWELL, A., SHENNAN, S. y THOMAS, M. G., 2010. «Demography and variation in the accumulation of culturally inherited skills». En M. J. O'Brien y S. J. Shennan (eds.), Innovation in Cultural Systems. Contributions from Evolutionary Anthropology, MIT Press, London, pp. 137-160.

QUERBES, A., VAESEN, K., y HOUKES, W. N., 2014. «Complexity and demographic explanations of cumulative culture». PLoS ONE, 9(7), e102543-1/9. https://doi. org/10.1371/journal.pone. 0102543

READ, D., 2006. «Tasmanian knowledge and skill: maladaptive imitation or adequate technology?». American Antiquity, 71, 164-184. https://doi. org/10.2307/40035327

READ, D., 2009. «The misuse of a mathematical model: the Tasmanian case (reply to Henrich's response)». UCLA: Human Complex Systems. http://dx.doi. org/10.2139/ssrn.2805822

READ, D., y LEBLANC, S. A., 2003. «Population Growth, Carrying Capacity, and Conflict». Current Anthropology, 44, 59-85. https://doi.org/10.1086/344616

RICHERSON, P. J., 2013. «Group size determines cultural complexity». Nature, 503, 351-352.

RICHERSON, P., BOYD, R. y BETTINGER, R., 2009. "Cultural Innovations and Demographic Change». Human biology, 81, 211-35. https://doi. org/10.3378/027.081.0306

SHENNAN, S., 2001.«Demography and culturalinnovation:amodelanditsimplications for the emergence of modern human culture». Cambridge Archaeological Journal, 11(01), 5-16. https://doi.org/10.1017/S0959774301000014

SHENNAN, S., 2015. «Demography and Cultural Evolution». En R.A. Scott y S.M. Kosslyn (eds.), Emerging Trends in the Social and Behavioral Sciences: An Interdisciplinary, Searchable, and Linkable Resource. Hoboken, NJ: John Wiley \& Sons, pp. 1-14.

SOLLAS, W. J., 1911. Ancient Hunters and their modern representatives. London: Macmillan.

STERELNY, K., 2020. «Demography and cultural complexity». Synthese, 1-24.

STRAUS, L. G., 2009. "Has the notion of 'transitions' in Paleolithic prehistory outlived its usefulness? The European record in wider context». En M. Camps y P. Chauhan (eds.), Sourcebook of Paleolithic Transitions. New York: Springer, pp. 3-18.

VAESEN, K., COLlARD, M., COSGROVE, R., y ROEBROEKS, W., 2016. «Population size does not explain past changes in cultural complexity». Proceedings of the National Academy of Sciences, 113(16), e2241-e2247. https:// doi.org/10.1073/pnas.1520288113 
ZILHÃO, J., 2007. «The Emergence of Ornaments and Art: An Archaeological Perspective on the Origins of "Behavioral Modernity"». Journal of Archaeological Research, 15, 1-54. https://doi.org/10.1007/s10814-006-9008-1

Alvaro Moreno Marin es Doctorando de la Universidad de Málaga.

Lineas de investigación

Filosofía de la biología, Evolución cultural, Teoría de la herencia dual

Correo electrónico: alvaromoma@gmail.com 
